\title{
Towards a System of Enhanced Transparency
}

\section{of Medical Curriculum}

\author{
Martin Komenda ${ }^{1}$, Daniel Schwarz ${ }^{1}$, Ladislav Dušek ${ }^{1}$ \\ ${ }^{1}$ Institute of Biostatistics and Analyses, Masaryk University, Brno, Czech Republic
}

\begin{abstract}
Introduction: A correctly compiled and balanced curriculum across medical fields is an essential prerequisite for medical education. Just as in other fields, it is vividly evident in medicine that the overall structure and lesson content are not ideal and it is quite common for the overlap between theoretical and clinical subjects to be either too extensive or, on the contrary, rather insufficient. The issue of curriculum innovation has been addressed in many medical and health care fields by different academic institutions, as proclaimed in the analysis of the current global situation. The need for a computer-aided management system providing a transparent overview of the curriculum at specific faculties has been duly emphasized.

Aim: An original methodology for managing the changes during optimization of the medical curriculum in tertiary education is introduced in the paper, together with its conceptual data model.
\end{abstract}

Methods: According to the conclusions of the Bologna Process, which promotes outcome-based education, the theoretical basis is oriented to the new paradigm of teaching and learning.

Results: We introduce the all-in-one web-oriented platform covering all elements linked to global curriculum management and providing sufficient information for the curriculum designers.

Conclusion: A new powerful and robust system for the management, visualization and analysis of curriculum should pinpoint specific learning imperfections and potential overlaps across the chosen field of study. It brings useful and beneficial information for students, teachers as well as the faculty management.

\section{Keywords}

Medical curriculum, outcome-based approach, computeraided management system.

\section{EJBI 2013; 9(3):9-16}

recieved: July 15, 2013

accepted: October 31, 2013

published: November 20, 2013

\section{Introduction}

Medical students face specific challenges compared to university students of other disciplines. The reason is that their future job does not allow any gaps in the knowledge obtained during the studies and that any error in their medical practice may have fatal consequences. The need for guaranteed and high-quality education involving predefined curricula covering the corresponding scope of input knowledge and skills required in subsequent practice has been gaining momentum. Medical universities compile their curricula so as to ensure that they cover all steps essential for the students to obtain employment later on. The students must fulfil all duties such as the successful completion of compulsory and optional courses and the final state exam, only to prepare for attestation to obtain professional qualification for employment as a physician.
A correctly compiled and balanced curriculum across medical fields is an essential prerequisite for medical doctor education. A suitable combination of theoretically focused courses and a clinical teaching base is certainly the key to a successful draft curriculum.

As in other fields and faculties, it is also evident in medicine that the overview of the lesson structure and content is not ideal and it often happens that the overlap between theoretical and clinical subjects is either too large or, on the contrary, rather insufficient. As modern information technologies have seen rapid development in the last decades, modern internet technologies can be utilized to develop an infrastructure or applications that would not only eliminate the poor transparency of the curricula but also help improve its construction as such.

The introduction of new approaches to learning, the exponential growth of Internet usage and the advent of 
the World Wide Web have the potential to change the face of higher education [1]. The Bergen ministerial conference of the Bologna Process in May 2005 discussed reforms pertaining to the degree structures, credit transfer, quality assurance and curricular development, which are transforming the European Higher Education Area. The European tertiary education systems are undergoing radical restructuring in line with the objectives defined by the Bologna Process. The design and structure of new curricula constitute significant processes of change and require cooperation and coordination [2. In the midst of these developments lies an interesting opportunity for applying new technologies providing an interface for collaborative curriculum content management, including graphical representation of the available data.

These systems will facilitate institutional decisionmaking activities related to the creation of a well-balanced curriculum. The entire concept is oriented to the new paradigm according to conclusions of the Bologna process, which introduces outcome-based education. This performance-based approach at the cutting edge of curriculum development offers a powerful and appealing way of reforming and managing medical education. The emphasis is on the product - what sort of doctors are we producing - rather than on the educational process [3]. Outcome-based education has emerged as a priority for curriculum planners striving to align with the ever demanding societal needs. The CanMEDS initiative of The Royal College of Physicians and Surgeons of Canada has introduced the implementation of a national, needsbased, outcome-oriented, competency framework that sets out the knowledge, skills and abilities specialist physicians need for better patient outcomes. The framework is based on the seven roles that all physicians need to have, to be better doctors: Medical Expert, Communicator, Collaborator, Manager, Health Advocate, Scholar, and Professional. This multifaceted approach has enabled a large-scale curriculum change for outcome-based education 4, 5.

\subsection{Outcome-Based Approach}

Learning outcomes are arguably best viewed as a fundamental building block of the Bologna educational reforms and bring more transparency to higher education systems. They have the reputation of rather mundane and prosaic tools, yet it is this basic underpinning function that makes them so significant. It is important that there should be no confusion about their role, nature and significance, or the educational foundations of the Bologna process will be undermined. Learning outcomes have applications at three distinct levels: (i) the local level of the individual higher education institution (for course units/modules, programmes of study and qualifications); (ii) the national level (for qualifications frameworks and quality assurance regimes); and (iii) internationally (for wider recognition and transparency purposes). Learning outcomes and "outcome-based approaches" have a strong impact on curriculum design, teaching, learning and assessment, as well as quality assurance [2].

The use of learning outcomes implies a fundamental paradigm shift in curriculum design for many European institutions offering higher education. The adoption of learning outcomes means a shift of emphasis from the content (what staff teach) to outcome (what a learner is expected to know, understand and/or be able to do), or expressed in other words, a clear shift from a teacher-centred to a learner-centred perspective. These issues are closely related to the teaching and learning methods as well as student workload. Defining the learning outcomes (instead of teaching inputs) promotes the idea of the teacher as a facilitator of learning and recognizes that a great part of learning takes place outside the classroom.

It promotes the idea of learners who are actively involved in the planning and management of their own learning activities and who assume more responsibility for it [6]. S. Adam defined in [7] that a learning outcome is a written statement of what the successful student/learner is expected to be able to do at the end of the module/course unit, or qualification. The key aspect that each of the definitions has in common is the desire for more precision and consideration as to what exactly a learner acquires in terms of knowledge and/or skills when he or she successfully completes the period of learning. Modern information and telecommunications technologies offer the opportunity to revolutionize the way we provide education [8]. When using ICTs (Information and Communication Technologies) for education, the temptation is often to focus more on the technology and less on the learners and instructors, often to the detriment of the education quality [9]. The combination of appropriate computer technologies and methodical approach (e.g. outcome-based approach) can fundamentally improve the entire education process.

\section{ICT Tools for Curriculum Management}

A recommended curriculum could be identified through content analysis of curriculum documents and interviews with individuals responsible for teaching [10]. The question is how can one make the whole process of curriculum harmonization easier and more effective? The quantity of electronic education content in the today's information society is steadily increasing. Thus, academic institutions require new innovative web-based tools for user-friendly creation, effective organization and clear visualization of outcome-based curriculum data. The true power of visualization tools is that they can provide a broader view of the whole curriculum. Apart from the aforementioned functionalities, the key and obviously necessary task rests in appropriate data visualization for easier understanding and further analysis. The term visualization represents a set of technical means and methods allowing clear and unambiguous graphical presentation of 


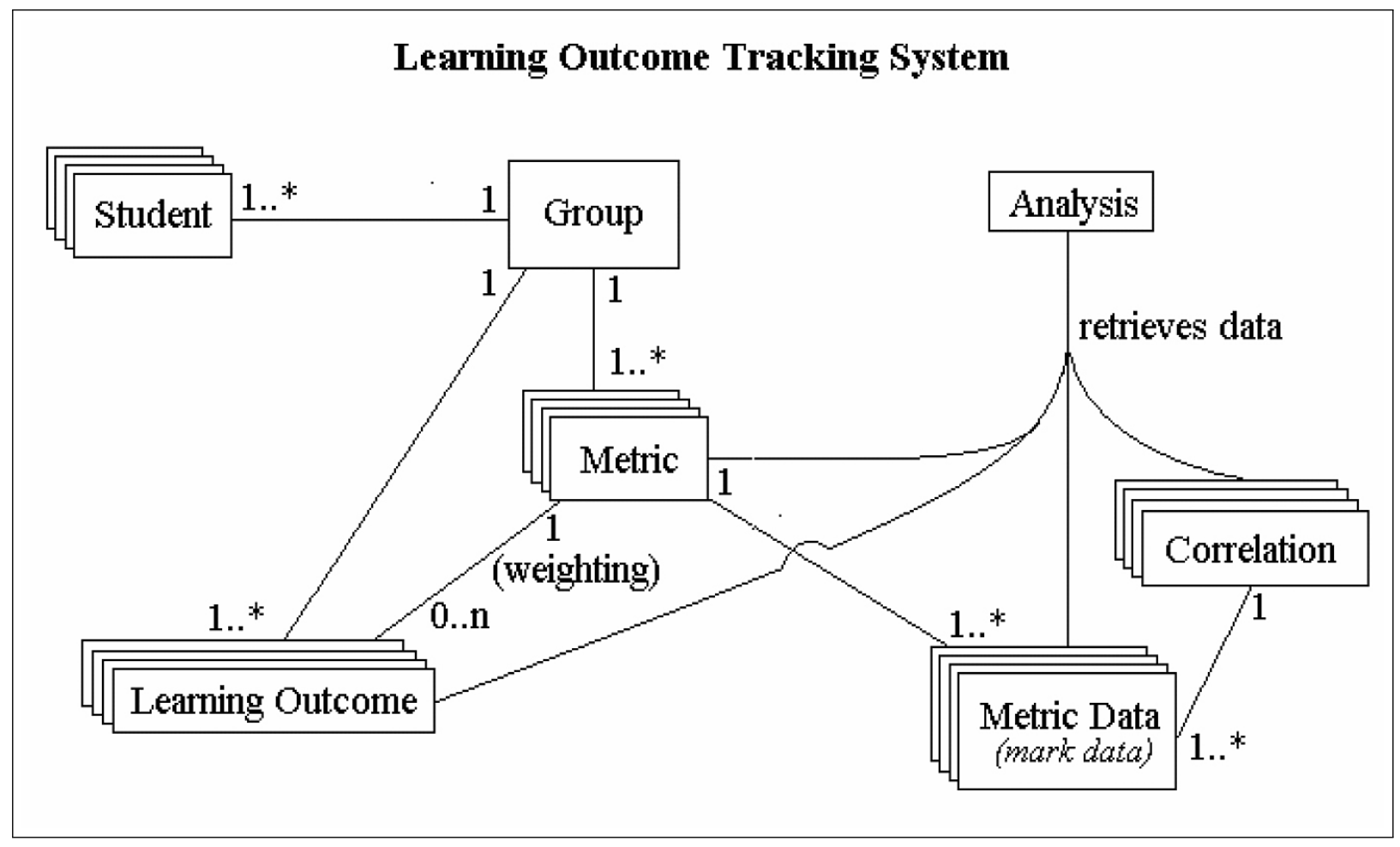

Figure 1: Relationship among the components for a single group instance in LOTS [13].

the data. The data stands in hidden relationships, its identification being the principal task of visual analysis that can be made with the integration of computer-based interactive tools and techniques based on cognitive, perceptual design principles [1].

There has been limited discussion on the original information technologies supporting outcome-based ideas. There are only a few available and published solutions, which have been developed for making educational outcome-based data accessible to the target group - students and teachers. In [12] A. Huang has presented an integrated outcome assessment application, for instance the design of a database to accumulate learner performance output by means of an outcome assessment application and storing it as learner profiles. These profiles could then serve as valuable input to produce customized learning content or to conduct overall performance evaluation. Y. Mong et al. 13 have described the web-based application LOTS (Learning Outcome Tracking System), which provides overall management of the learning outcomes and access for both the student and the teachers. In brief, LOTS consists of six components, namely group, metric, learning outcome, incident, correlation and analysis.

Fig. 1 illustrates the relationship among the components for a single group instance. The group includes student and group administration, where users can manage students and groups in the database. A group can be a course, a degree programme, or a subset of students. A great benefit is the integration of LOTS with an existing LMS (Learning Management System) envi- ronment. LOTS includes also several types of basic analysis with graphical output presentations (typically bar charts) for users to exploit the advantages of outcomebased education.

The "generic" electronic portfolio called ePortfolio 14 is an application, which is being used to support the evidencing of learning outcomes and to facilitate personal development planning. In modular courses, portfolios may provide focus on programme-level as well as module-specific learning outcomes. The whole process may help students become better at relating what they have learned to the requirements of teachers. The ePortfolio has been developed using robust platform-independent open source tools. In the design phase, some of the potential "value-added" features, which an IT approach can bring compared to paper-based portfolios, were considered, i.e. being highly customizable with multiple structures and views, easier cross-referencing, being searchable, integration with virtual learning environments, etc. A number of generic tools have been developed for supporting personal development planning, i.e. a reflective learning diary, outcomes/skills log, records of meetings with tutors, SWOT matrix and an Action Planning tool. The ePortfolio framework also allows sharing of specific content with supervisors, peers and others, with the facility for viewers to add formative comments.

Dynamic Learning Maps (DLM) has provided a novel way to navigate and engage with the curriculum and support learning. Curriculum drivers included the longstanding need for maps to aid the understanding of complex curricula in Medicine, and for promoting "cross- 


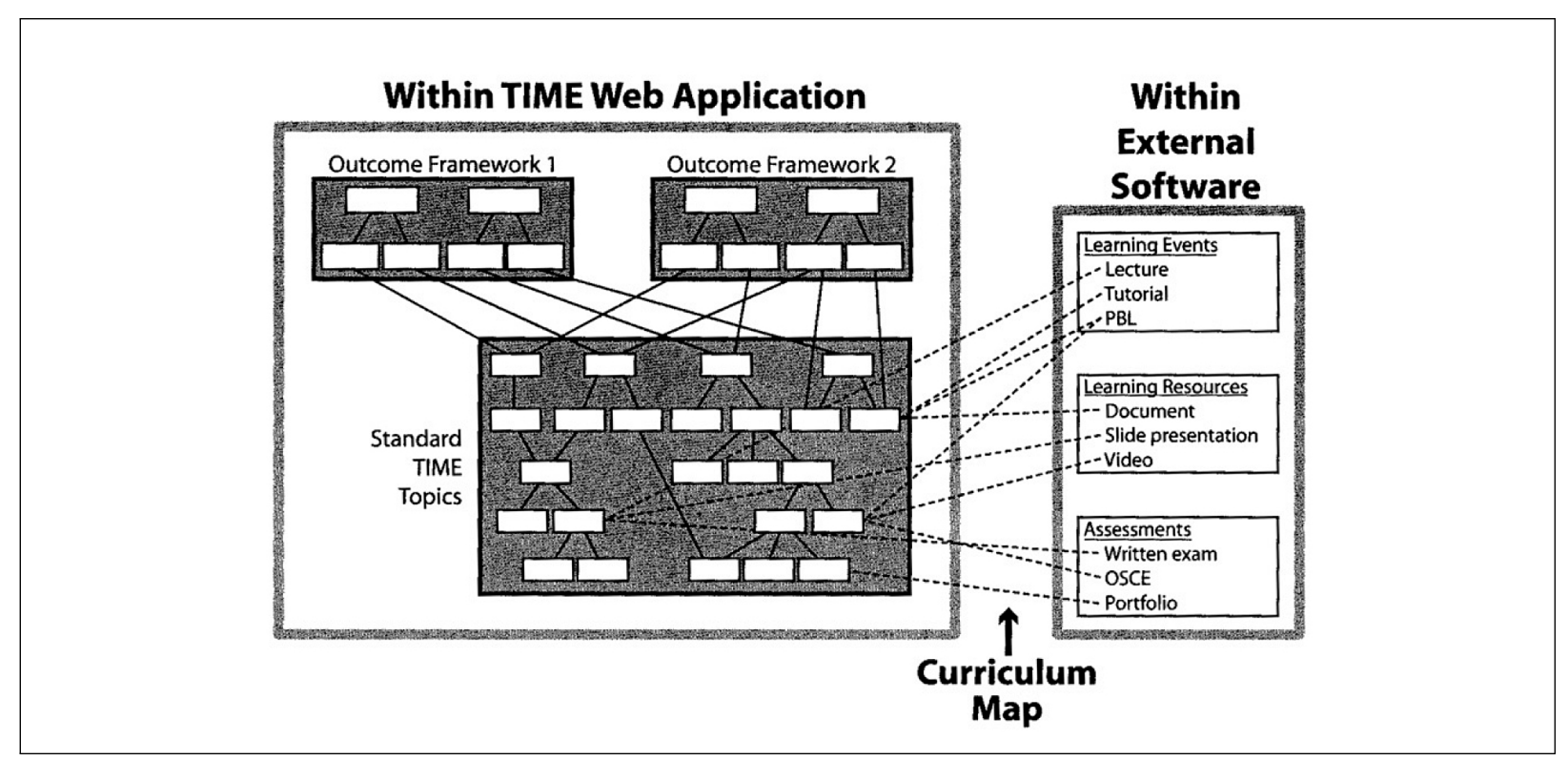

Figure 2: TIME includes Outcomes, Topics and Objectives [19].

modular" learning in modular programmes. DLM has been designed to be flexible and extensible and can be used for a range of different pedagogic/educational purposes. This web-based tool utilizes existing technologies and standards to provide access to information from curriculum databases, ePortfolios and other established sources. This approach provides a unique resource for learners, teachers and curriculum managers alike by providing a detailed and convenient way of accessing the formal and informal curriculum. DLM is an interactive tool, which can be aligned with an institution's Teaching and Learning Strategy, while at the same time it supports a wide range of requirements for specific programmes. There are two main views: "tree view" (hierarchical text) and "MindMap". For example, the maps can display and make links between granular curriculum information and overarching transferable skills frameworks and/or programme-level outcomes. This novel concept has been well received by learners, teachers and curriculum staff and has had considerable impact both at the Newcastle University and within the broader CETIS community (the Centre for Educational Technology and Interoperability Standards). DLMs took a rapid development/action research style approach, with emphasis on formative evaluation involving substantial input from students, teachers and other stakeholders. Open-source software was used for DLM development, in particular the Python-based rapid development framework Django, MySQL databases, jQuery and other Javascript libraries and the Freemind Flash browser [15, 16].

S. Kabicher et al have presented a sophisticated approach, the use of visual modelling within an interactive online environment (ActiveCC Web) for collaborative design, implementation and visualization of the curriculum structure and content. ActiveCC, an abbreviation for Active Curriculum for Computer Science, is an e-learning project at the Faculty of Computer Science, University of Vienna. The project aims to provide an insight into the structure and implementation of the new computer science curriculum to support the teaching staff in coordinating their course contents and to support the instructors at the faculty in obtaining an overview of the curriculum structure. It was technically based on the Cooperative Environment Web Services platform, a web service-oriented architecture for cooperation and learning, which offers a wiki module including the functionality of directly writing graph visualization code into the wiki. Using the graph visualization tool, the temporal arrangement of modules and dependency links among modules are visually modelled to show the modules' location and role within the curriculum. This approach offers an easily accessible and intuitively editable virtual space to facilitate collaboration on the curriculum content, provides a transparent view of the curriculum's structure and an insight into the module and course implementations, content and pedagogical methods [17, 18].

One of the options for describing content related to the curriculum is special taxonomy. T. G. Willett et al. have published a paper introducing TIME (Topics for Indexing Medical Education), a hierarchical taxonomy of topics relevant to medical education. The content and structure of the topics within TIME was developed in consultation with medical educators and librarians at several Canadian medical schools. As a web application, TIME can contain three types of items that are distinct but related to each other in hierarchical manner. It can contain Outcomes, which we operationally define as culminating demonstrations of learning at the end of medical training; Topics, which describe the content (subjects) of medical education; and Objectives, which we define as the more specific expectations associated with the courses or learning opportunities. 


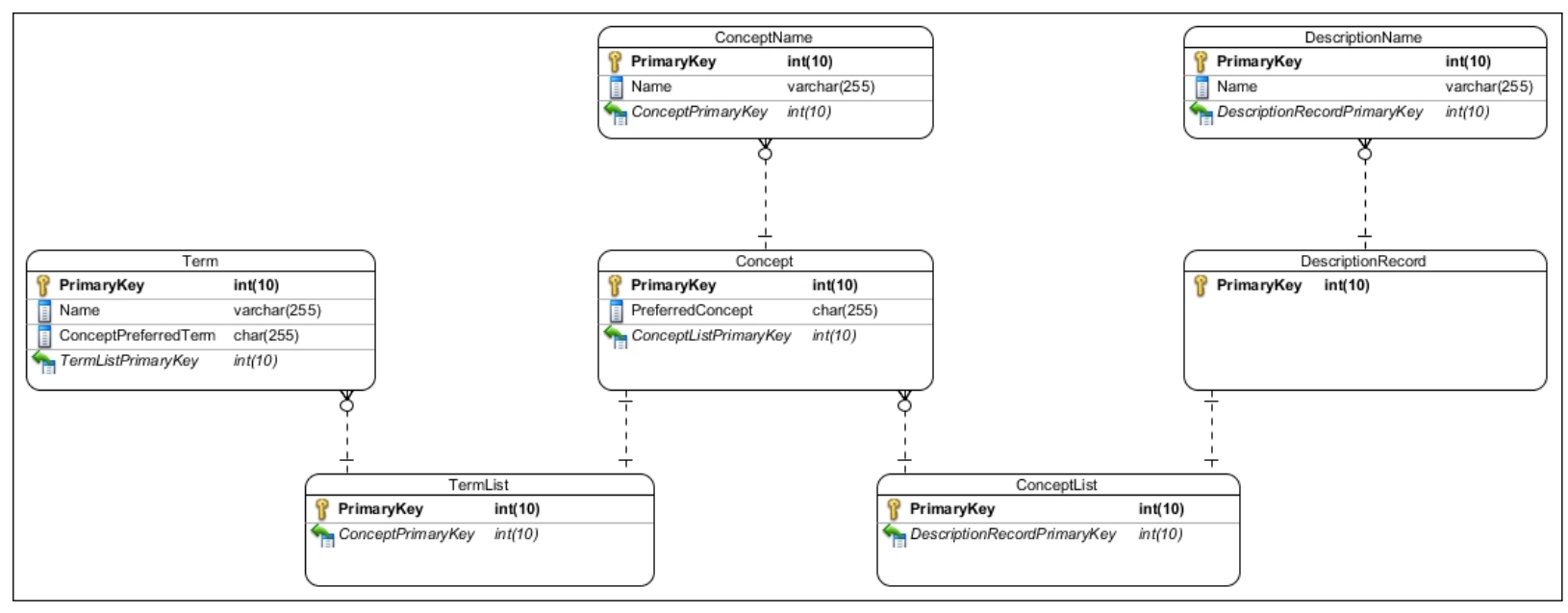

Figure 3: Conceptual data model covers all essential attributes for curriculum description using MeSH thesaurus.

The TIME is primarily intended to be a generic index for medical education. It is meant to be applicable to curriculum maps, as metadata for tagging reusable learning objects, as a classification for assessment objects, as a means of linking Objectives to Outcomes for a medical school, or potentially for any other indexing purpose in undergraduate medical education. The application itself does not enable mapping or indexing. This simply weboriented tool allows the user to browse topics, create new outcomes and objectives, and link these items to the topics. The entire TIME content and structure can then be exported, via XML (eXtensible Markup Language), to external applications and used as an index for curriculum mapping [19]. Today, unfortunately, the TIME application is not available and cannot be viewed via the recommended web address and follow-up development was probably terminated.

\section{Computer-Aided Management of Curriculum: Design}

The idea of connection topics via the TIME web application is not the most suitable solution for complex and easy optimization of a curriculum, but it brings new inspiration for further development. Most importantly, we need an all-in-one web-oriented service, which covers all phases of the optimization process. This means an interface for data creation and editing, a user-friendly tool for transparent browsing supporting fast and accurate search, and a technology for graphical visualization of the curriculum relations. As stated above, there does not appear today to be any solution for how to parametrically describe, effectively manage and visualize clearly and indepth the curriculum and all related education metadata in one system. This is the primary motivation for developing a new original technology for optimizing a curriculum, which supports an outcome-based approach. A similar solution based on the aforementioned parametric description of curriculum, including all appropriate metadata details (i.e. attributes of learning outcomes, units and objects), has not been widely seen.

Existing solutions that have been published are focused on the curriculum only from a certain perspective, offering the agenda together with selected functionalities and making the effort to provide them to students and teachers of the respective institution in a transparent format. However, we have yet to see a complex instrument that would cover all elements associated with global curriculum optimization, including a detailed parametric description down to the level of the learning units, and that would be linked to the learning objects. The main benefit of the development is a new concept resting on the following functionalities - a concept that has not yet been implemented in similar solutions.

- A proposal of an original medical curriculum optimization methodology across clinical and theoretical fields based on elaborate curriculum parameterization by using the outcome-based paradigm $[3,7,13$.

- A proposal of a formal database metadata arrangement describing the general medical curriculum independent of subsequent implementation.

- Integration of the Medical Subject Headings (MeSH) thesaurus [20] for standardized work with key words. As a specific field taught in the Czech language shall be optimized, a dictionary was selected that is standardized in English and offers Czech translation with updates on annual basis.

- A proposal of an elaborate access right system using a central verification mechanism through the Shibboleth technology [21] to allow potential future extension of access to the user level among academic community members. 


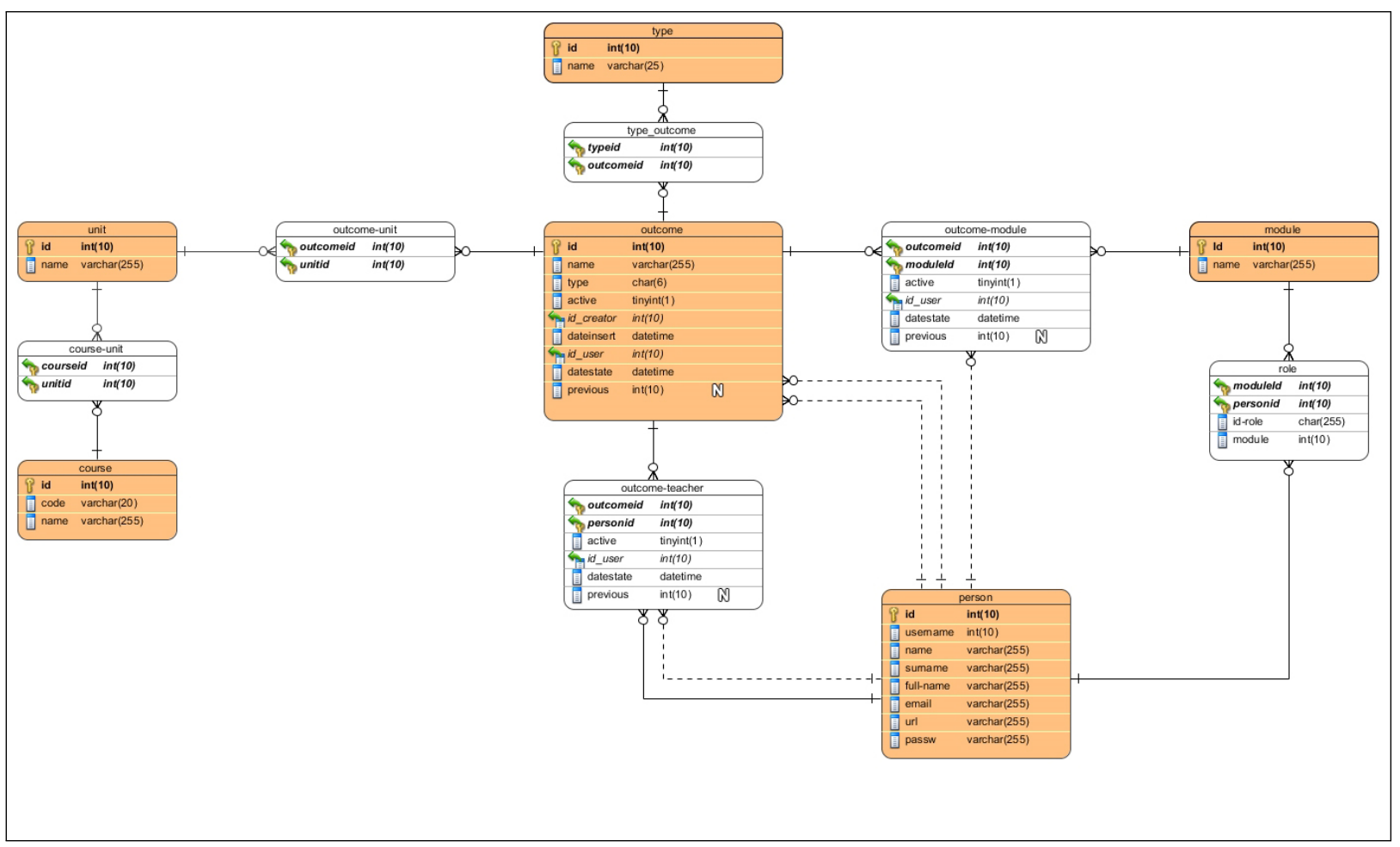

Figure 4: Entity relation data model represents key attributes of the proposed platform.

\section{Results}

The development of a new curriculum optimization technology and general methodology description are among the key attributes of our effort. The outcomebased approach opens the possibility of redefining the curriculum structure and properties in the form of a parametric description. The learning outcome is a summary of the requirements for graduates from the general medicine field, i.e. the list of knowledge and skills which the student should have upon completion of his/her studies, and signals the information essential for eventual work as a doctor. The organization of this data and its correlation will be provided in the curriculum data model that can be implemented without any restrictions within any database technology. The new methodology describes the different phases of the optimization process by using the platform to be developed. Its objective is to make all work of the user more efficient regarding the creation, editing and control mechanisms in the form of deep content inspection.

The architecture of the designed platform comprises two sections: FrontOffice and BackOffice. FrontOffice represents the interface to be used for presenting the content to the end user. The content of this section will be freely available only to the target group of users, i.e. students and teachers. BackOffice is the second platform component to be used by administrators and editors and is intended as the interface for adding and editing static content. Another feature is the adoption of the Czech version of the MeSH thesaurus where the objective is to standardize key words relating to the metadata stored in the database. The actual key words are defined and structured in many forms and there is a growing need for their unification with respect to the international framework. The bio-medicine dictionary MeSH in the English language has been published since 1960 by the US National Library of Medicine. The Czech translation of this thesaurus is being prepared by the Czech National Medical Library, which issues annual updates. The dictionary contains 26142 entries with over 54000 links 20]. Following a contractual agreement with the Czech National Medical Library, MeSH will also be used for the purposes of the emerging curriculum optimization platform. The main requirements for standardized dictionary integration are regular updates of the Czech mutation, which MeSH fulfils as the only solution available. No other language mutations are foreseen at the moment, but a possible change should not bring too many complications in terms of the proposed structure.

Besides the key words defined in an internationally recognized and standardized format, the learning unit description also features the so-called essential terms for which no dictionary exists. The database of essential terms contained in the field that the students come across during the six years of their study programme and which the graduates should be familiar with, will be fed by teachers and guarantors of studies. A unique dictionary of essential terms in medical studies will emerge and offer another view of the sophisticated and vast curriculum. To ensure at least partial standardization (quasistandardization), users will be assisted by autocompletion as they enter words. The autocompletion will display 
expressions corresponding with the text already typed existing words or corresponding items already defined by past authors. After the final clean-up, which cannot be fully automated, one of the outcomes (and hence the benefit) is the still non-existent dictionary of essential expressions from the general medicine field of study.

The draft entity relation model (see Fig. 4) is the basis for possible implementation in practice. It describes, in depth, the key entities and defines the relationships between them so that all selected links can later be displayed in a transparent manner. The entire optimization process is split into several dependent phases allowing efficient mapping of the curriculum of the respective field or specialization of study. A characteristic tool is recommended for each phase to give the end user - the teacher - the possibility of intuitively creating/browsing certain content. All tools have been developed based on real requirements and are the practical manifestation of how the method can be implemented when correct IT is applied. Unlike the platform being developed, the application of this method is fully independent of the information and communication technologies and of the focus (the selection of the field of study that will be optimized).

\section{Discussion}

Since the platform will serve users as a tool to oversee their curricula, attention will be focused in the future also on more efficient and fast search agenda. A language morphology analyzer is planned to be employed in the overall framework of the designed platform. For the Czech language, services of the original Majka analyzer [22] will be used. The objective is to integrate the script that will process different input chains into root forms and store this information in a database along with a link to the related metadata. If the user enters a search expression, then all words containing the root of the search expression will be displayed thanks to this functionality. As the current full-text search does not provide any reliable method of working with word forms for the Czech language, this processing method is an interesting and desirable solution. The root forms of words will also play an important role in the implementation of analytical and visualization tools. This domain will consist of data mining, data pre-processing (lemmatization, lexical categorization, creation of stop words list, generation of term frequency vector), data analysis (clustering, similarity measures), and visualization (graphs and networks). Additionally, selected methods of natural language processing and machine learning will be used for effective identification of information rich data relations and for simpler and easier way of understanding curriculum structure.

\section{Conclusions}

This approach is aimed to be primarily used for a global curriculum audit and it should determine the learn- ing imperfections and potential overlaps across the chosen field of study. Building a powerful and robust system for the management, visualization and analysis of the curriculum could extend the basic learning outcome concept. What will such an approach mean for the student? It will provide clear information about what knowledge shall be obtained over the six years, what topics will be on the agenda, what fields will be covered repeatedly and what subjects are linked to the studies. For teacher's parametric description will mean an easier way of how to clearly describe their lessons and how to browse the curriculum data of all available courses at the medical faculty according to the pre-defined parameters. For the faculty management, this overview will represent a practical view of the teaching. It will provide clear and comprehensible data about who teaches what and in what scope, if an overlap is desirable, what is taught in clinical and theoretical fields and if the overall teaching pattern is correct or whether restructuring is necessary. The platform will also bring objective information used to define the new structure of the curriculum.

\section{Acknowledgements}

Martin Komenda and Daniel Schwarz were supported from the grant project "MEFANET clinical reasoning" reg. n.: CZ.1.07/2.2.00/28.0038, which is funded by the European Social Fund and the state budget of the Czech Republic.

\section{References}

[1] R. M. Harden and I. R. Hart, "An international virtual medical school (IVIMEDS): the future for medical education?," Med. Teach., vol. 24, no. 3, pp. 261-267, May 2002.

[2] R. Keeling, "The Bologna Process and the Lisbon Research Agenda: the European Commission's expanding role in higher education discourse," Eur. J. Educ., vol. 41, no. 2, pp. 203-223, 2006.

[3] R. M. Harden, J. R. Crosby, and M. H. Davis, "AMEE Guide No. 14: Outcome-based education: Part 1?An introduction to outcome-based education," Med. Teach., vol. 21, no. 1, pp. $7-14,1999$.

[4] J. R. Frank and D. Danoff, "The CanMEDS initiative: implementing an outcomes-based framework of physician competencies," Med. Teach., vol. 29, no. 7, pp. 642-647, 2007.

[5] J. R. Frank and M. D. Bernard Langer OC, "Collaboration, communication, management, and advocacy: teaching surgeons new skills through the CanMEDS Project," World J. Surg., vol. 27, no. 8, pp. 972-978, 2003.

[6] S. Adam, "Using learning outcomes - A consideration of the nature, role, application and implications for European education of employing 'learning outcomes' at the local, national and international levels," University of Westminster, Edinburgh, 2004.

[7] S. Adam, "An introduction to learning outcomes," Consid. Nat., 2006.

[8] B. Barnes and C. Friedman, "Using technology in continuing professional development," in The continuing professional 
development of physicians, vol. 2003, Chicago: The continuing professional development of physicians, pp. 205-220.

[9] J. Sargeant, "Medical education for rural areas: Opportunities and challenges for information and communications technologies," J. Postgrad. Med., vol. 51, no. 4, pp. 301-7, Dec. 2005.

[10] D. T. Stern, "Practicing What We Preach? An Analysis of the Curriculum of Values in Medical Education," Am. J. Med., vol. 104, no. 6, pp. 569-575, erven 1998.

[11] D. Randjelovic and B. Popovic, "Visual analytics tools and theirs application in social networks analysis," in Telecommunications Forum (?FOR), 2011 19th, 2011, pp. $1340-1343$

[12] A. H. Huang, "A framework of educational technology applications and development opportunities.," J. Comput. Inf. Syst., vol. 41, no. 3, pp. 54-58, 2001.

[13] Y. Mong, M. Chan, and F. K. H. Chan, "Web-based outcomebased teaching and learning - An experience report," in Advances in Web Based Learning - Icwl 2007, vol. 4823, H. Leung, F. Li, R. Lau, and Q. Li, Eds. Berlin: Springer-Verlag Berlin, 2008, pp. 475-483.

[14] S. Cotterill, "Design, implementation and evaluation of a 'generic' ePortfolio: the Newcastle experience," in Proc. ePortfolio 2004 .

[15] S. Ball, "Dynamic Learning Maps -Final Project Report." 2009 .
[16] P. Horner, S. Cotterill, J. Peterson, and G. Skelly, "Dynamic Learning Maps," in Learning Forum London 2010-Internet of Subjects Forum ePortfolio-Key Competencies-Identity London, 5-7 July 2010, Savoy Place Published by EIfEL ISBN 2-9524576-9-7., p. 98

[17] S. Kabicher and M. Derntl, "Visual Modelling for Design and Implementation of Modular Curricula," Z. Für Hochschulentwicklung, 2008

[18] S. Kabicher, M. Derntl, and R. Motschnig-Pitrik, "ActiveCC - A Collaborative Framework for Supporting the Implementation of Active Curricula," J. Educ. Multimed. Hypermedia, vol. 18, no. 4, pp. 429-451, 2009.

[19] T. G. Willett, K. C. Marshall, M. Broudo, and M. Clarke, "TIME as a generic index for outcome-based medical education," Med. Teach., vol. 29, no. 7, pp. 655-659, Jan. 2007.

[20] "Fact SheetMedical Subject Headings (MeSH®)." [Online]. Available: http://www.nlm.nih.gov/pubs/factsheets /mesh.html. [Accessed: 12-Jul-2012]

[21] M. Needleman, "The Shibboleth Authentication/Authorization System," Ser. Rev., vol. 30, no. 3, pp. 252-253, 2004.

[22] P. Šmerk, "Fast Morphological Analysis of Czech," RASLAN 2009 Recent Adv. Slavon. Nat. Lang. Process., p. 13, 2009. 